\title{
1 Advances in de novo strain design using integrated systems and synthetic 2 biology tools
}

3 Chiam $\mathrm{Yu} \mathrm{Ng}{ }^{1}$, Ali Khodayari ${ }^{1}$, Anupam Chowdhury ${ }^{1}$ and Costas D. Maranas

Address: Department of Chemical Engineering, The Pennsylvania State University, University Park, PA, USA

${ }^{1}$ Joint first authors

\begin{abstract}
Recent efforts in expanding the range of biofuel and biorenewable molecules using microbial production hosts have focused on the introduction of non-native pathways in model organisms and the bioprospecting of non-model organisms with desirable features. Current challenges lie in the assembly and coordinated expression of the (non-)native pathways and the elimination of competing pathways and undesirable regulation. Several systems and synthetic biology approaches providing contrasting top-down and bottom-up strategies, respectively, have been developed. In this review, we discuss recent advances in both in silico and experimental approaches for metabolic pathway design and engineering, with a critical assessment of their merits and remaining challenges.
\end{abstract}

\section{Introduction}

Microbial production has the unique advantage over chemical catalysis in that it can co-opt thousands of enzymes finely tuned by nature and leverage the host's biological processes for cofactor regeneration, catalytic machinery assembly/disassembly and housekeeping functions. Advancement in metabolic engineering has increased the range of bio-based chemical products in microbial hosts, including therapeutics such as artemisinin [1], bioplastic precursors such as 1,4-butanediol [2], and biodiesel fatty esters and fatty acids [3]. Despite several success stories, only few metabolic engineering products achieve performance metrics that currently merit commercialization [4].

Increasing demands on maximizing production potential have highlighted the importance of developing tools that can identify more efficient pathways, both native and heterologous to a given host, from a (often given) substrate to the target chemical. Subsequently, metabolic intervention strategies are drawn to reconfigure the host metabolism for channeling additional flux towards the selected pathway and eliminating carbon and redox losses towards undesirable products, followed by the construction and evaluation of the strains. However, there are several challenges to the successful implementation of this design-build-test-learn loop (Figure 1). Enzymes are sensitive to temperature and $\mathrm{pH}$ and cannot be universally expressed in all hosts with a controllable rate of expression [5]. Another challenge lies in the successful implementation of computational predictions due to incomplete/erroneous modeling descriptions as well as the inability to precisely modulate gene expression to match model predictions. Furthermore, the current capacity to generate combinatorial variants far exceeds the throughput of screening. 
In this review, we focus on recent advances in systems and synthetic biology for synthetic metabolic pathway design and optimization. We first describe the recently developed computational tools for identifying de novo biosynthetic pathways. Next, we discuss computational stoichiometry-based and kinetic-based approaches for strain optimization. Finally, we discuss the recently developed synthetic biology and genome engineering techniques for synthetic pathway and network engineering.

\section{Pathway prospecting for synthetic routes}

Synthetic pathways from a source metabolite to a target chemical must satisfy a number of performance criteria such as (i) maximal use of native reactions [6] (Figure 1b, in orange), (ii) minimal number of reaction steps or equivalently total enzymatic load [7] (Figure 1b, in blue), (iii) maximization of product yield [8] (Figure 1b, in grey), (iv) cofactor balance in the overall pathway [6], and (v) thermodynamic feasibility of the overall pathway and individual steps [9]. A priori assessment of whether these criteria are met requires knowledge of the metabolism of the host organism (e.g., codified as a genome-scale network (Figure 1a)) and other database resources. Optimizing native pathways generally requires less effort as both catalytic components and regulatory structures are already in place [5]. In contrast, expression of non-native reactions is more complex but the upside is that they can in many cases significantly improve the yield of target products [10].

A number of pathway-prospecting tools have been developed recently (Table 1a) leveraging advances in computational power and availability of well-curated databases of metabolic reactions. Elementary Flux Mode (EFM) derived approaches relying on Linear Programming (LP) formulations can now be extended to genome-scale models and comprehensive reaction databases to search for de novo pathways. Variations of this approach have been implemented for reconfiguring novel amino acid synthesis pathways in $E$. coli [7], engineering hosts for biomass-coupled chemical production (e.g., SSDesign [11]) and designing novel pathways for $\mathrm{CO}_{2}$-fixation [12]. Alternatively, the computational intractability of EFMs in searching from thousands of reaction candidates [7] can be ameliorated by using graph-based tools for pathway design [13]. These tools can now rank pathways based on product yield [14] and cost of transcription and translation (e.g., DESHARKY [15]), as well as prevent selection of thermodynamically infeasible intermediate reactions (e.g., Metabolic Tinker [9]). In addition, atom-mapping information have also been incorporated to trace the fate of individual atoms (especially carbon) that filters out non-carbon transferring paths (e.g., Carbon Flux Path [16] ).

Recent tools in pathway design use a set of "reaction rules" instead of lists of reactions to predict novel pathways without being restricted to previously catalogued reactions in nature. A retrosynthetic algorithm selects the intermediate metabolites between source and target chemical by satisfying the rules of chemical transformation defined by the set of reaction operators (e.g., BNICE [17], XTMS [18]). For example, in the recent GEM-Path approach [6], Biochemical Reaction Operators (BROs) serve as reaction templates for conversion of metabolites. Using an iterative algorithm to trace back from the target molecule one reaction at a time, metabolites are assigned to the BROs using a scoring mechanism based on how similar a metabolite is to the existing host metabolome. A reaction is accepted if it is present in a curated database, or is similar enough to an existing enzyme in the database to catalyze the putative reaction. The algorithm proceeds to identify the previous reaction in the linear pathway terminating when a metabolite present in the host metabolome is identified.

Despite enormous progress over the past few years, available pathway design procedures are generally restricted to only (near) linear routes from the source to the target metabolite. Linear pathway designs generally miss cyclic networks with potential for higher efficiency (both carbon and energy) of production. In addition, by restricting the degrees of freedom to just the source and target metabolite, the 
identification of alternative co-reactants/co-products combinations are ignored. While post-processing efforts restore stoichiometry-balance of pathways [6], this may lead to designs with suboptimal carbon and energy efficiencies. Compatibility of a heterologous pathway with the metabolic host of interest is also often not adequately addressed at the design stage [19]. While some of the procedures minimize the number of heterologous enzymes [6,20], or choose enzymes phylogenetically closest to the host [21], there is no guarantee that the synthetic pathway would be host compatible. In addition, existing computational procedures do not directly assess the toxicity potential of intermediate metabolites. As more toxicity data for model organisms is collected (e.g., PanDaTox [22]), toxicity prediction tools (e.g., EDGE [23]) would increasingly become more commonplace in scoring synthetic pathways. Likewise, kinetic properties of the enzymes in the pathway would increasingly be queried to find the most active routes to the target chemical (e.g., DESHARKY [15]).

\section{Modeling-driven pathway engineering}

Once the designed pathway is introduced in the host strain, the metabolic fluxes need to be re-apportioned towards the target product (Figure 1c). Several optimization-based computational techniques have been developed to achieve this aim [24] (Table 1b). The scope of these approaches has been expanded through the use of synthetic biology tools. These predictive tools comprised of stoichiometry-only approaches [25], kinetic models [26] or hybrid combinations thereof [27] make quantitative predictions on metabolism upon metabolic interventions.

A number of efforts have integrated non-native pathways into the production host metabolic model by simply expanding the stoichiometric matrix using Flux Balance Analysis (FBA) techniques. For example, Proportional Flux Forcing (PFF) [28] was developed to explore the effect of substrate competition upon insertion of non-native genes using the GDLS algorithm. This is achieved by forcing a fixed fraction of the flux passing through the substrate into the alternative heterologous pathways formed by the introduced genes. This procedure was used for enhancing free fatty acid production in E. coli. In another effort, Yim et al [29] integrated a biopathway prediction algorithm with computational strain design protocols to identify synthetic pathways producing non-native products from common metabolic intermediates in $E$. coli. They first constructed an ensemble of 10,000 pathways producing 1,4-butanediol (14BDO) from mixed sugar streams. The best engineering strategies were then identified using the OptKnock algorithm [30] improving the yield of 14BDO production for the two best-ranked synthetic pathways.

Stoichiometry-based approaches are limited to steady-state conditions and are generally unable to describe the rate of reaction in terms of the underlying pool of metabolite concentrations and enzyme abundances. Therefore, the identified metabolic engineering strategies may not be implementable. For example, for a suggested up-regulation the corresponding enzymatic activity and metabolite concentrations may not be reachable and/or physiologically allowable. These shortcomings can potentially be addressed by kinetic models that directly track both enzyme levels and concentrations [31].

The application of kinetic-based models in synthetic biology, however, is still hampered by a number of challenges, chief among which are the paucity, in vivo applicability and universality of kinetic parameter data. In an effort to alleviate this problem, Farasat et al proposed SEAMAPs to build a kinetic model for a given modular synthetic pathway [32]. They used RBS Library Calculator to design minimal number of experiments, which varied expression of each enzyme in the pathway over 10,000-fold, to parameterize the kinetic model of neurosporene production pathway in $E$. coli. In another effort, a kinetic model was developed to identify the rate-limiting step of an in vitro ATP-free synthetic pathway for production of hydrogen from pretreated biomass sugars [33]. 
Successful implementation of kinetic expressions to guide metabolic interventions requires that regulatory interactions at the substrate, transcriptional, translational and post-translational levels are adequately captured [34]. Using metabolite concentration and enzyme activity as model variables, significant progress in the integration of substrate level regulatory interactions in kinetic models has been achieved [35]. Implementation of transcription level regulatory interactions in stoichiometry-based models is limited to Boolean representations or introduction of ad hoc constraints to shrink the flux ranges in concert with transcriptomic and proteomic data. This posture generally assumes that a positive correlation exists between metabolic flux and gene expression levels though there exists ample counter-examples [36]. Generally, the predictive accuracy of these approaches is highly condition dependent [37]. The scope of kinetic models can be further expanded to integrate transcription-level regulatory events. This can potentially be achieved using phenomenological Hill equations or partition functions to describe the rate of mRNA synthesis from a given promoter in terms of transcription factor (TF) activities [38]. This could ultimately enable the integration of transcriptional regulatory events with models of metabolism.

Kinetic-based modeling approaches show promise in capturing the dynamic behavior of metabolic pathways and regulatory interactions. Integration of such models with system-level omics data and computational metabolic engineering tools provides an avenue for understanding and subsequently optimizing the function of synthetic pathways. Robust model parameterization in response to genetic/environmental perturbations remains difficult. Many efforts are currently underway towards resolving this challenge by proposing more efficient optimization approaches [39], reducing parameters search space by structural analysis [40] and efficient sampling approaches [41].

\section{Synthetic biology and genome engineering tools for implementation of pathway predictions}

Ideally, the engineered strain should match as close as possible the desired flux distribution predicted through metabolic modeling. This requires among other considerations precise and reliable control of gene transcription and mRNA translation. Various genetic parts including promoters, RBSs, terminators, TFs and small regulatory RNA (sRNA) have been extensively characterized, offering an unprecedented range of parts for engineering gene expression. However, the performance of genetic parts is often nonconserved across different contexts (e.g. host, genetic, media) thus requiring performance re-assessment in the desirable conditions [42]. Alternatively, several computational techniques have been developed for designing context-specific sequences (e.g., RBS Calculator [43]) and selecting the optimum combination (e.g., OptCircuit [44]) of genetic parts (Table 1c).

Optimization of gene expression involves a vast design sequence space (i.e., $4^{\mathrm{n}}$, where $\mathrm{n}$ is the length of a transcription unit). In addition, several parameters can affect gene expression such as codon usage, tRNA availability, secondary structures, presence of RNases binding sites, internal Shine-Dalgarno sequences and repeats [45]. Some of these parameters are contradictory thus confounding the task of codon selection. For example, rare codons instead of common codons at the N-terminal tend to reduce secondary structures thus increasing the rate of protein translation [46]. A number of gene design tools attempt to integrate all these sometimes conflicting requirements [45], albeit with limited experimental verification. An alternative approach is to rely on high-throughput gene synthesis technology to generate large codonusage variants set for a particular protein and then screen for high-expression variants, thus circumventing the need-to-know all design rules [47].

In addition to ensuring proper expression of all genes, the expression level of the entire synthetic pathway must also be carefully tuned to prevent imbalance in cellular resources (e.g., biomass precursors, proteins and redox equivalents [48]) and accumulation of toxic metabolites [49]. Combinatorial approaches geared towards optimizing the expression of all enzymes use various techniques ranging from targeting a rational 
selection of genes to random editing at a genome-scale. Combinatorial DNA assembly techniques such as Gibson Assembly [50] and DNA Assembler [51] are commonly used to fuse genes or operons with libraries of regulatory parts (e.g., promoter [52-54], RBS [55] and copy number [55]). When the expression of a large number of genes must be manipulated, they are often partitioned into separate modules based on their functions to reduce the search space. Notably, Ajikumar et al varied the expression of methylerythritol-phosphate (MEP) pathway and taxadiene synthesis modules by changing their promoter and copy number [56]. Despite exploring just a small fraction of the entire combinatorial space, their combinatorial variants achieved up to 15,000-fold change in taxadiene production [56]. On a larger scale, the Klebsiella oxytoca nitrogen fixation gene cluster (103 parts) was refactored by employing a combination of combinatorial design and assembly approach [5].

Advances in high-throughput genome engineering techniques have accelerated the construction of large strain libraries (Figure 1e). Methods such as MAGE [57] and synthetic sRNAs [58] directly target tens of pre-selected genes with high specificity, whereas other techniques such as gTME [59], SCALEs [60] and TRMR [61] first generate libraries of strains with randomized genome-wide mutations and subsequently perform selection to identify the genotype conferring the desired traits. Both approaches complement one another, as the latter can be used for identifying subsets of genes required for wider range of expression tuning by the former. Recently, CRISPR-Cas9 (or dCas9) system has emerged as a versatile tool for multiplex genome engineering [62]. This system requires the design of highly orthogonal guide RNA(s) with minimal off-target activity. Collectively, these approaches are already capable of rapidly generating large combinatorial libraries, however, lack of high-throughput assays currently limits their applications to phenotypes with colorimetric (e.g., carotenoids production) or growth-based assays (e.g., metabolite tolerance).

As for other difficult to detect metabolites, intracellular biosensors have led to a number of success stories [63]. TF-based biosensors couple the expression of reporter proteins (typically fluorescent protein or antibiotic resistance marker) to the level of a metabolite of interest, enabling isolation of desired mutants via fluorescence-activated cell sorting (FACS) or positive growth selection [64,65]. Raman et al recently fine-tuned their biosensors so as only cells that produce target chemicals above a certain threshold would survive [66]. They performed FBA to identify target genes for MAGE genome engineering and then employed their biosensors to select for high producers, resulting in 36-fold and 22-fold improvement of naringenin and glucaric acid production, respectively.

While it is important to map desired traits to their genotype, sequencing and characterizing the entire combinatorial libraries remains cost-prohibitive. Microarray technology has been previously employed for parallel genotype-phenotype mapping of large gene knockout and overexpression libraries [60,61]. Recently, the tracking combinatorial engineered libraries (TRACE) method combines DNA assembly and next-generation sequencing for simultaneous genotype mapping of individual cells within a large combinatorial population [67]. TRACE was then employed to efficiently track both combinatorial diversity and evolution trajectory of a MAGE population [67].

All of these techniques have significantly improved pathway engineering and the strain construction process. In addition, they provide useful datasets for validation and refinement of computational tools and stoichiometric and kinetic metabolic models. For example, multiplex gene-knockout techniques can rapidly generate multiple knockout mutants to validate synthetic lethality predictions [68]. The refined model can then be used for predicting genetic manipulation strategies with better accuracy.

\section{Future perspectives}


With an ever expanding ability to construct, screen and characterize large mutant strain libraries, emphasis will likely shift on the ability to analyze large heterogeneous datasets for guiding the discovery of improved variants. Both machine learning inspired approaches that look for patterns in "big data" and predictive frameworks that seamlessly integrate different layers of biological processes would be needed. Moving beyond the scope of biological functions catalogued in nature, prospecting tools such as BNICE [17] and GEM-Path [6] could pro-actively be used to pinpoint desirable enzyme substrate and/or cofactor activity changes by harnessing enzyme plasticity. This has the potential for the discovery of more direct routes to target chemicals bypassing enzymatic or regulatory bottlenecks. Furthermore, the systematic discovery and pro-active elimination of undesirable secondary enzymatic functions that could drain carbon flux away from the main product could help shorten the strain design cycle [69]. Both tasks require the reliable re-design of enzymes either using de novo [70] or evolutionary techniques [71]. Several computational design techniques have been proposed for improving enzyme turnover number, substrate specificity, reduced allosteric inhibition, etc. [72-74], but reliable protein design remains elusive [75]. Improvements in our ability to model and predict the outcome of biological processes and networks coupled with falling DNA synthesis costs and efficient DNA assembly tools are bringing closer to fruition the dream of the design and assembly of synthetic production hosts uniquely tailored for bio-product biosynthesis.

\section{Acknowledgements}

22 This study was supported by the United States Department of Energy (DOE) grant DE-SC0008091. 


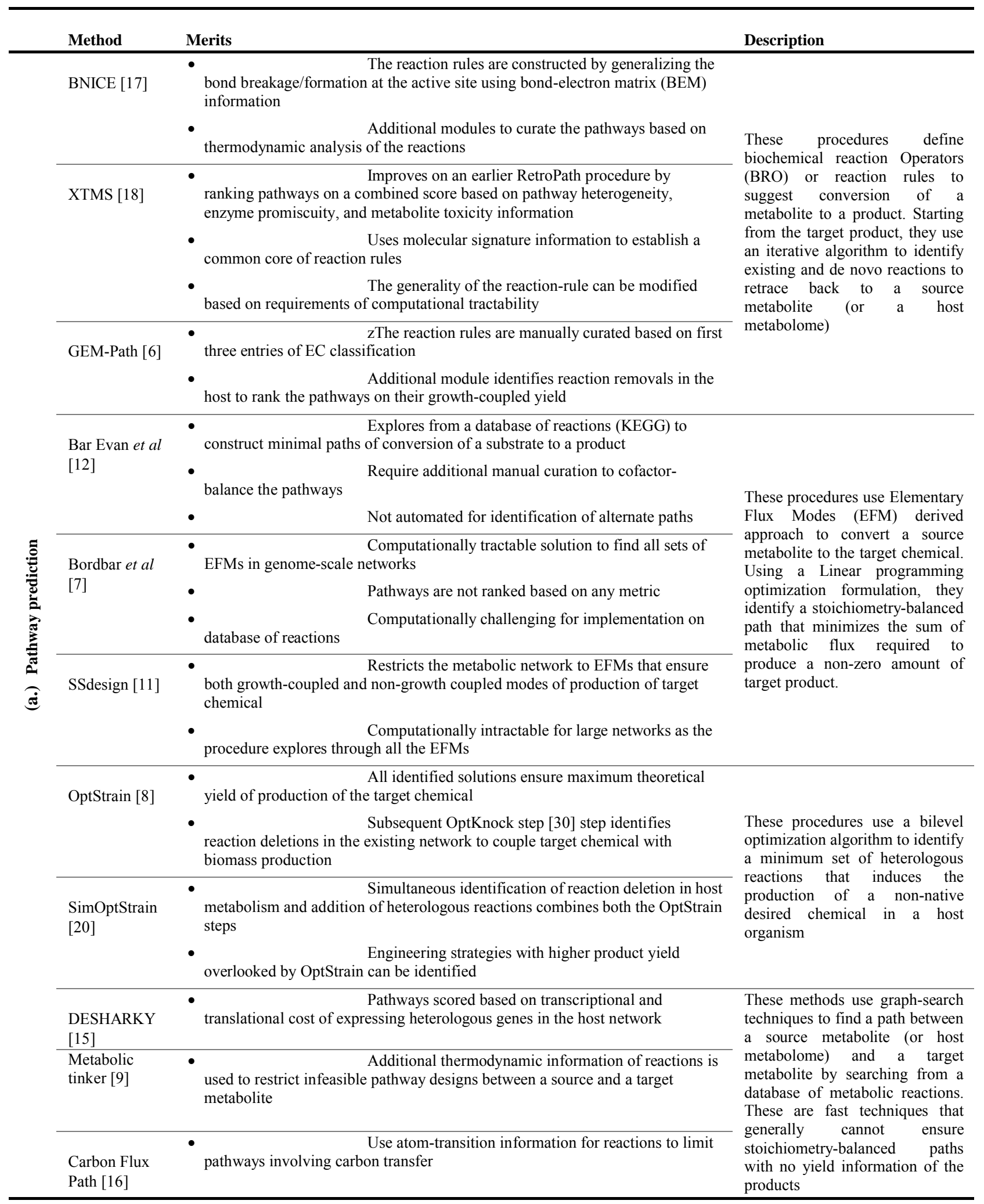




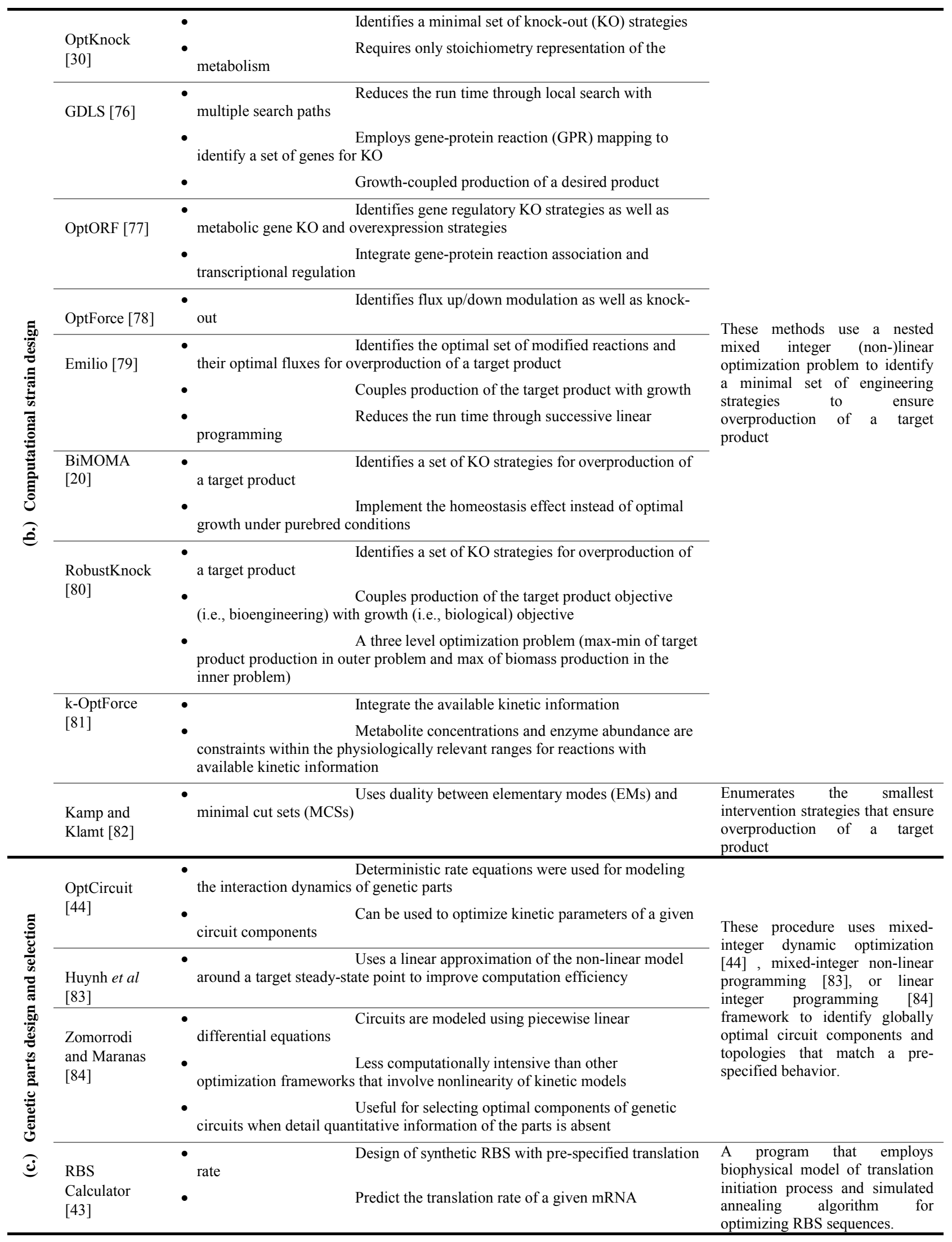


Figure Legend

Figure 1. Pictorial overview of computational and experimental techniques for strain development and pathway engineering.

\section{References}

1. Paddon CJ, Westfall PJ, Pitera DJ, Benjamin K, Fisher K, McPhee D, Leavell MD, Tai a, Main a, Eng $\mathrm{D}$, et al.: High-level semi-synthetic production of the potent antimalarial artemisinin. Nature 2013, 496:528-532.

2. Yim H, Haselbeck R, Niu W, Pujol-Baxley C, Burgard A, Boldt J, Khandurina J, Trawick JD, Osterhout RE, Stephen R, et al.: Metabolic engineering of Escherichia coli for direct production of 1,4-butanediol. Nature chemical biology 2011, 7:445-452.

3. Xu P, Li L, Zhang F, Stephanopoulos G, Koffas M: Improving fatty acids production by engineering dynamic pathway regulation and metabolic control. Proceedings of the National Academy of Sciences of the United States of America 2014, 111:1-6.

4. Van Dien S: From the first drop to the first truckload: commercialization of microbial processes for renewable chemicals. Curr Opin Biotechnol 2013, 24:1061-1068.

5. Smanski MJ, Bhatia S, Zhao D, Park Y, L BAW, Giannoukos G, Ciulla D, Busby M, Calderon J, Nicol $\mathrm{R}$, et al.: Functional optimization of gene clusters by combinatorial design and assembly. Nat Biotechnol 2014, 32:1241-1249.

• 6. Campodonico MA, Andrews BA, Asenjo JA, Palsson BO, Feist AM: Generation of an atlas for commodity chemical production in Escherichia coli and a novel pathway prediction algorithm, GEM-Path. Metab Eng 2014, 25:140-158.

This work introduces the Gem-Path procedure, a retrosynthetic algorithm of designing de novo paths from the metabolome of a host organism to a non-native target product using pre-formulated reaction operators. Combined with stoichiometry balance and thermodynamics feasibility modules, this approach has a post-processing step for engineering the host for growth-coupled product.

• 7. Bordbar A, Nagarajan H, Lewis NE, Latif H, Ebrahim A, Federowicz S, Schellenberger J, Palsson BO: Minimal metabolic pathway structure is consistent with associated biomolecular interactions. Mol Syst Biol 2014, 10:737.

This work describes a Mixed Integer Linear Programming (MILP) optimization formulation that can successfully identify all the shortest, linearly independent pathways in a genome-scale network. Along with discovering hidden transcriptional regulation controlling pathway flux, this method also identified novel minimal routes for amino acid synthesis in E. coli and S. cerevisiae.

8. Pharkya P, Burgard AP, Maranas CD: OptStrain: a computational framework for redesign of microbial production systems. Genome Res 2004, 14:2367-2376.

9. McClymont K, Soyer OS: Metabolic tinker: an online tool for guiding the design of synthetic metabolic pathways. Nucleic Acids Res 2013, 41:e113.

10. Lan EI, Liao JC: ATP drives direct photosynthetic production of 1-butanol in cyanobacteria. Proc Natl Acad Sci U S A 2012, 109:6018-6023.

11. Toya Y, Shiraki T, Shimizu H: SSDesign: Computational metabolic pathway design based on flux variability using elementary flux modes. Biotechnol Bioeng 2015, 112:759-768.

12. Bar-Even A, Noor E, Lewis NE, Milo R: Design and analysis of synthetic carbon fixation pathways. Proc Natl Acad Sci U S A 2010, 107:8889-8894. 
13. Liu F, Vilaca P, Rocha I, Rocha M: Development and application of efficient pathway enumeration algorithms for metabolic engineering applications. Comput Methods Programs Biomed 2015, 118:134-146.

14. Yousofshahi M, Lee K, Hassoun S: Probabilistic pathway construction. Metab Eng 2011, 13:435444.

15. Rodrigo G, Carrera J, Prather KJ, Jaramillo A: DESHARKY: automatic design of metabolic pathways for optimal cell growth. Bioinformatics 2008, 24:2554-2556.

-16. Pey J, Planes FJ, Beasley JE: Refining carbon flux paths using atomic trace data. Bioinformatics 2014, 30:975-980.

This work extends an earlier Carbon Flux path approach to introduce atom-transition details between metabolites in reactions to identify paths that only involve carbon transfer between a source and a target metabolite.

17. Hatzimanikatis V, Li C, Ionita JA, Henry CS, Jankowski MD, Broadbelt LJ: Exploring the diversity of complex metabolic networks. Bioinformatics 2005, 21:1603-1609.

18. Carbonell P, Parutto P, Herisson J, Pandit SB, Faulon JL: XTMS: pathway design in an eXTended metabolic space. Nucleic Acids Res 2014, 42:W389-394.

19. Chatsurachai S, Furusawa C, Shimizu H: An in silico platform for the design of heterologous pathways in nonnative metabolite production. BMC Bioinformatics 2012, 13:93.

20. Kim J, Reed JL, Maravelias CT: Large-scale bi-level strain design approaches and mixed-integer programming solution techniques. PLoS One 2011, 6:e24162.

21. Tamura K, Peterson D, Peterson N, Stecher G, Nei M, Kumar S: MEGA5: molecular evolutionary genetics analysis using maximum likelihood, evolutionary distance, and maximum parsimony methods. Mol Biol Evol 2011, 28:2731-2739.

22. Amitai G, Sorek R: PanDaTox: a tool for accelerated metabolic engineering. Bioengineered 2012, 3:218-221.

-23. Wagner A, Zarecki R, Reshef L, Gochev C, Sorek R, Gophna U, Ruppin E: Computational evaluation of cellular metabolic costs successfully predicts genes whose expression is deleterious. Proc Natl Acad Sci U S A 2013, 110:19166-19171.

This work develops an Expression-Dependent gene Effects (EDGE) procedure in which a MILP optimization problem predicts whether the overexpression of an in vivo (or non-native) gene would be deleterious/advantageous/ to the growth potential of a host organism. This approach was used to predict and verify "toxic genes" in several organisms and suggest a procedure of distinguishing cancerous cells from healthy one due to repressed expression of these "toxic genes".

24. Copeland WB, Bartley BA, Chandran D, Galdzicki M, Kim KH, Sleight SC, Maranas CD, Sauro HM: Computational tools for metabolic engineering. Metab Eng 2012, 14:270-280.

25. Zomorrodi AR, Suthers PF, Ranganathan S, Maranas CD: Mathematical optimization applications in metabolic networks. Metab Eng 2012, 14:672-686.

26. Almquist J, Cvijovic M, Hatzimanikatis V, Nielsen J, Jirstrand M: Kinetic models in industrial biotechnology - Improving cell factory performance. Metab Eng 2014, 24:38-60.

- 27. Khodayari A, Chowdhury A, Maranas CD: Succinate Overproduction: A Case Study of Computational Strain Design Using a Comprehensive Escherichia coli Kinetic Model. Front Bioeng Biotechnol 2014, 2:76.

This study uses k-OptForce procedure to identify the minimal interventions that improve the yield of succinate production using a hybrid kinetic-stoichiometry model of E. coli metabolism.

28. Ip K, Donoghue N, Kim MK, Lun DS: Constraint-based modeling of heterologous pathways: application and experimental demonstration for overproduction of fatty acids in Escherichia coli. Biotechnol Bioeng 2014, 111:2056-2066. 
29. Yim H, Haselbeck R, Niu W, Pujol-Baxley C, Burgard A, Boldt J, Khandurina J, Trawick JD, Osterhout RE, Stephen R, et al.: Metabolic engineering of Escherichia coli for direct production of 1,4-butanediol. Nat Chem Biol 2011, 7:445-452.

30. Burgard AP, Pharkya P, Maranas CD: Optknock: a bilevel programming framework for identifying gene knockout strategies for microbial strain optimization. Biotechnol Bioeng 2003, 84:647-657.

31. Matsuoka Y, Shimizu K: Current status and future perspectives of kinetic modeling for the cell metabolism with incorporation of the metabolic regulation mechanism. Bioresources and Bioprocessing 2015, 2:1-19.

32. Farasat I, Kushwaha M, Collens J, Easterbrook M, Guido M, Salis HM: Efficient search, mapping, and optimization of multi-protein genetic systems in diverse bacteria. Mol Syst Biol 2014, 10:731.

33. Rollin JA, Martin Del Campo J, Myung S, Sun F, You C, Bakovic A, Castro R, Chandrayan SK, Wu $\mathrm{CH}$, Adams MW, et al.: High-yield hydrogen production from biomass by in vitro metabolic engineering: Mixed sugars coutilization and kinetic modeling. Proc Natl Acad Sci U S A 2015.

34. Heinemann M, Sauer U: Systems biology of microbial metabolism. Curr Opin Microbiol 2010, 13:337-343.

35. Khodayari A, Zomorrodi AR, Liao JC, Maranas CD: A kinetic model of Escherichia coli core metabolism satisfying multiple sets of mutant flux data. Metab Eng 2014, 25:50-62.

36. Kremling A, Bettenbrock K, Gilles ED: A feed-forward loop guarantees robust behavior in Escherichia coli carbohydrate uptake. Bioinformatics 2008, 24:704-710.

37. Machado D, Herrgard M: Systematic evaluation of methods for integration of transcriptomic data into constraint-based models of metabolism. PLoS Comput Biol 2014, 10:e1003580.

• 38. Garcia HG, Phillips R: Quantitative dissection of the simple repression input-output function. Proc Natl Acad Sci U S A 2011, 108:12173-12178.

This work presents a thermodynamic model to quantify the level of gene expression as a function of regulatory

tuning variables using partition functions.

39. C. P, Miróa A, Guillén-Gosálbeza G, Sorribasc A, Alvesc R, Jiméneza L: Gobal optimization of hybrid kinetic/FBA models via outer-approximation. Computers \& Chemical Engineering 2015, 72:325-333.

•40. Lee Y, Lafontaine Rivera JG, Liao JC: Ensemble Modeling for Robustness Analysis in engineering non-native metabolic pathways. Metab Eng 2014, 25:63-71.

In this study, the Ensemble Modeling (EM) paradigm is integrated with continuation method for robustness analysis

(EMRA) of the (non-)native pathways.

41. Tan Y, Liao JC: Metabolic ensemble modeling for strain engineers. Biotechnol J 2012, 7:343-353.

42. Arkin AP: A wise consistency: engineering biology for conformity, reliability, predictability. Curr Opin Chem Biol 2013, 17:893-901.

43. Salis HM, Mirsky Ea, Voigt Ca: Automated design of synthetic ribosome binding sites to control protein expression. Nature biotechnology 2009, 27:946-950.

44. Dasika MS, Maranas CD: OptCircuit: an optimization based method for computational design of genetic circuits. BMC Syst Biol 2008, 2:24.

45. Gould N, Hendy O, Papamichail D: Computational tools and algorithms for designing customized synthetic genes. Frontiers in bioengineering and biotechnology 2014, 2:41.

46. Goodman DB, Church GM, Kosuri S: Causes and effects of N-terminal codon bias in bacterial genes. Science 2013, 342:475-479.

47. Quan J, Saaem I, Tang N, Ma S, Negre N, Gong H, White KP, Tian J: Parallel on-chip gene synthesis and application to optimization of protein expression. Nature biotechnology 2011, 29:449-452. 
48. Ng CY, Farasat I, Maranas CD, Salis HM: Rational design of a synthetic Entner-Doudoroff pathway for improved and controllable NADPH regeneration. Metab Eng 2015, 29:86-96.

49. Zelcbuch L, Antonovsky N, Bar-Even A, Levin-Karp A, Barenholz U, Dayagi M, Liebermeister W, Flamholz A, Noor E, Amram S, et al.: Spanning high-dimensional expression space using ribosome-binding site combinatorics. Nucleic Acids Res 2013, 41:e98.

50. Gibson DG, Young L, Chuang R-Y, Venter JC, Hutchison CA, Smith HO: Enzymatic assembly of DNA molecules up to several hundred kilobases. Nature methods 2009, 6:343-345.

51. Shao Z, Zhao $\mathrm{H}$, Zhao $\mathrm{H}$ : DNA assembler, an in vivo genetic method for rapid construction of biochemical pathways. Nucleic Acids Res 2009, 37:e16.

52. Lee ME, Aswani A, Han AS, Tomlin CJ, Dueber JE: Expression-level optimization of a multienzyme pathway in the absence of a high-throughput assay. Nucleic Acids Res 2013, 41:10668-10678.

53. Du J, Yuan Y, Si T, Lian J, Zhao H: Customized optimization of metabolic pathways by combinatorial transcriptional engineering. Nucleic Acids Res 2012, 40:e142.

54. Shao Z, Rao G, Li C, Abil Z, Luo Y, Zhao H: Refactoring the silent spectinabilin gene cluster using a plug-and-play scaffold. ACS Synth Biol 2013, 2:662-669.

55. Xu P, Gu Q, Wang W, Wong L, Bower AG, Collins CH, Koffas MA: Modular optimization of multi-gene pathways for fatty acids production in E. coli. Nat Commun 2013, 4:1409.

56. Ajikumar PK, Xiao WH, Tyo KE, Wang Y, Simeon F, Leonard E, Mucha O, Phon TH, Pfeifer B, Stephanopoulos G: Isoprenoid pathway optimization for Taxol precursor overproduction in Escherichia coli. Science 2010, 330:70-74.

57. Wang HH, Isaacs FJ, Carr Pa, Sun ZZ, Xu G, Forest CR, Church GM: Programming cells by multiplex genome engineering and accelerated evolution. Nature 2009, 460:894-898.

• 58. Na D, Yoo SM, Chung H, Park H, Park JH, Lee SY: Metabolic engineering of Escherichia coli using synthetic small regulatory RNAs. Nature biotechnology 2013, 31:170-174.

This work employs rationally designed synthetic small regulatory RNAs (sRNAs) for precise control of target gene expression levels. Using synthetic sRNAs, they improved the production of tyrosine by combinatorial knock-down of four genes across fourteen different $E$. coli strains. They further targeted over 130 genes, including essential genes, to optimize cadaverine production.

59. Alper H, Stephanopoulos G: Global transcription machinery engineering: a new approach for improving cellular phenotype. Metab Eng 2007, 9:258-267.

60. Lynch MD, Warnecke T, Gill RT: SCALEs: multiscale analysis of library enrichment. Nat Methods 2007, 4:87-93.

61. Warner JR, Reeder PJ, Karimpour-Fard A, Woodruff LB, Gill RT: Rapid profiling of a microbial genome using mixtures of barcoded oligonucleotides. Nat Biotechnol 2010, 28:856-862.

62. Jakočiūnas T, Bonde I, Herrgård M, Harrison SJ, Kristensen M, Pedersen LE, Jensen MK, Keasling JD: Multiplex metabolic pathway engineering using CRISPR/Cas9 in Saccharomyces cerevisiae. Metabolic Engineering 2015, 28:213-222.

63. Eggeling L, Bott M, Marienhagen J: Novel screening methods-biosensors. Curr Opin Biotechnol 2015, 35C:30-36.

64. Dietrich JA, Shis DL, Alikhani A, Keasling JD: Transcription factor-based screens and synthetic selections for microbial small-molecule biosynthesis. ACS Synth Biol 2013, 2:47-58.

65. Binder S, Schendzielorz G, Stabler N, Krumbach K, Hoffmann K, Bott M, Eggeling L: A highthroughput approach to identify genomic variants of bacterial metabolite producers at the single-cell level. Genome Biol 2012, 13:R40.

• 66. Raman S, Rogers JK, Taylor ND, Church GM: Evolution-guided optimization of biosynthetic pathways. Proc Natl Acad Sci U S A 2014, 111:17803-17808.

This work employed FBA-guided multiplex genome engineering and growth-coupled transcription factor-based biosensors to optimizse narigenin and glucaric acid biosynthetic pathway. 
•67. Zeitoun RI, Garst AD, Degen GD, Pines G, Mansell TJ, Glebes TY, Boyle NR, Gill RT: Multiplexed tracking of combinatorial genomic mutations in engineered cell populations. Nat Biotechnol 2015.

This study presented TRACE, an approach that perform genome mutation mapping of $>10^{5}$ individual cells from combinatorially engineered populations.

68. Suthers PF, Zomorrodi A, Maranas CD: Genome-scale gene/reaction essentiality and synthetic lethality analysis. Mol Syst Biol 2009, 5:301.

69. Guzman GI, Utrilla J, Nurk S, Brunk E, Monk JM, Ebrahim A, Palsson BO, Feist AM: Model-driven discovery of underground metabolic functions in Escherichia coli. Proc Natl Acad Sci U S A 2015, 112:929-934.

70. Rajagopalan S, Wang C, Yu K, Kuzin AP, Richter F, Lew S, Miklos AE, Matthews ML, Seetharaman $\mathrm{J}$, Su M, et al.: Design of activated serine-containing catalytic triads with atomic-level accuracy. Nat Chem Biol 2014, 10:386-391.

71. Smith MA, Romero PA, Wu T, Brustad EM, Arnold FH: Chimeragenesis of distantly-related proteins by noncontiguous recombination. Protein Sci 2013, 22:231-238.

72. Privett HK, Kiss G, Lee TM, Blomberg R, Chica RA, Thomas LM, Hilvert D, Houk KN, Mayo SL: Iterative approach to computational enzyme design. Proc Natl Acad Sci U S A 2012, 109:3790-3795.

73. Tinberg CE, Khare SD, Dou J, Doyle L, Nelson JW, Schena A, Jankowski W, Kalodimos CG, Johnsson K, Stoddard BL, et al.: Computational design of ligand-binding proteins with high affinity and selectivity. Nature 2013, 501:212-216.

74. Grisewood MJ, Gifford NP, Pantazes RJ, Li Y, Cirino PC, Janik MJ, Maranas CD: OptZyme: computational enzyme redesign using transition state analogues. PLoS One 2013, 8:e75358.

75. Pantazes RJ, Grisewood MJ, Maranas CD: Recent advances in computational protein design. Curr Opin Struct Biol 2011, 21:467-472.

76. Lun DS, Rockwell G, Guido NJ, Baym M, Kelner JA, Berger B, Galagan JE, Church GM: Largescale identification of genetic design strategies using local search. Mol Syst Biol 2009, 5:296.

77. Kim J, Reed JL: OptORF: Optimal metabolic and regulatory perturbations for metabolic engineering of microbial strains. BMC Syst Biol 2010, 4:53.

78. Ranganathan S, Suthers PF, Maranas CD: OptForce: an optimization procedure for identifying all genetic manipulations leading to targeted overproductions. PLoS Comput Biol 2010, 6:e1000744.

79. Yang L, Cluett WR, Mahadevan R: EMILiO: a fast algorithm for genome-scale strain design. Metab Eng 2011, 13:272-281.

80. Tepper N, Shlomi T: Predicting metabolic engineering knockout strategies for chemical production: accounting for competing pathways. Bioinformatics 2010, 26:536-543.

81. Chowdhury A, Zomorrodi AR, Maranas CD: k-OptForce: integrating kinetics with flux balance analysis for strain design. PLoS Comput Biol 2014, 10:e1003487.

82. von Kamp A, Klamt S: Enumeration of smallest intervention strategies in genome-scale metabolic networks. PLoS Comput Biol 2014, 10:e1003378.

83. Huynh L, Kececioglu J, Koppe M, Tagkopoulos I: Automatic design of synthetic gene circuits through mixed integer non-linear programming. PLoS One 2012, 7:e35529.

84. Zomorrodi AR, Maranas CD: Coarse-grained optimization-driven design and piecewise linear modeling of synthetic genetic circuits. European Journal of Operational Research 2014, 237:665-676. 


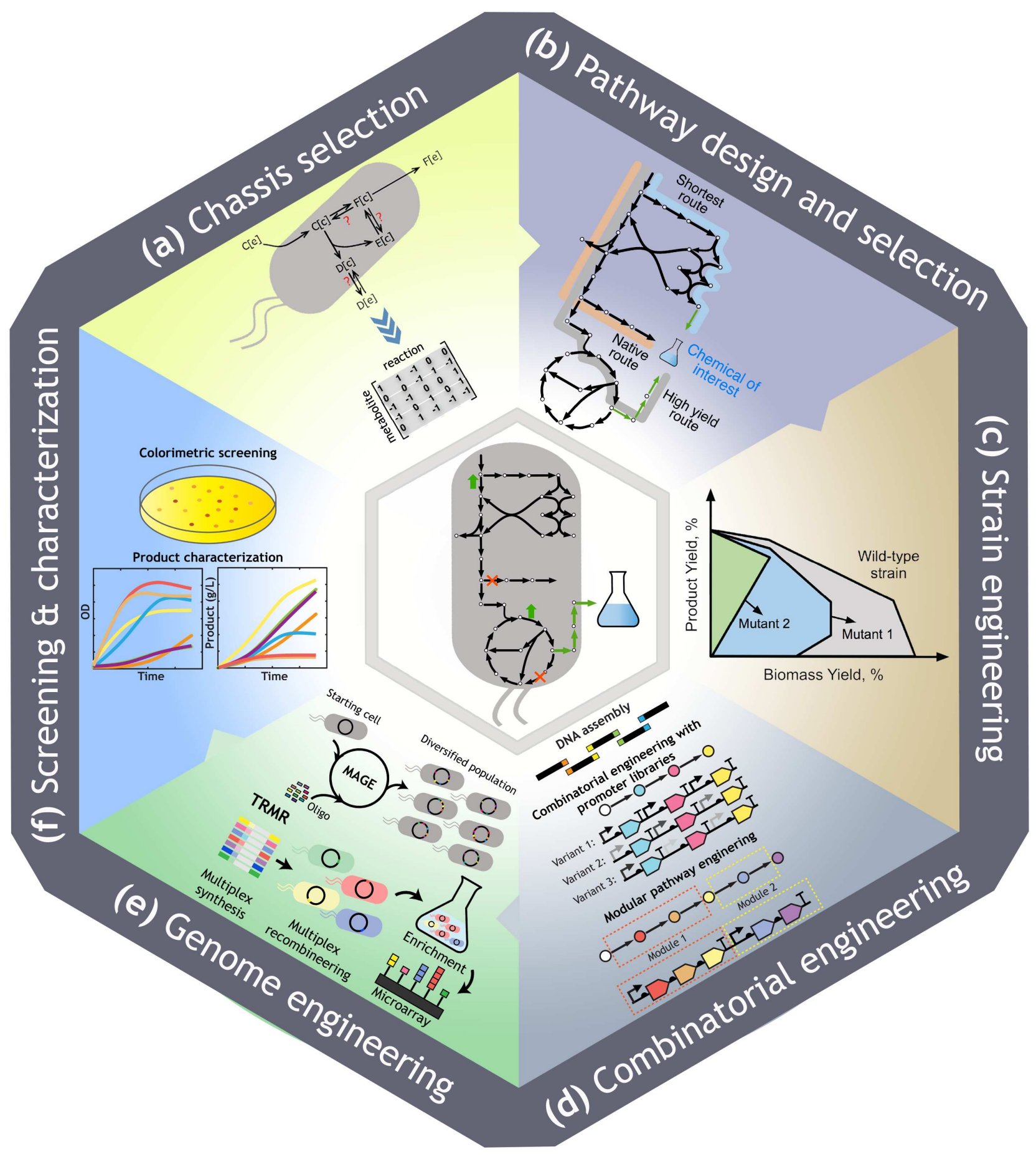

